\title{
Pharmacokinetic predictions and docking studies of substituted aryl amine-based triazolopyrimidine designed inhibitors of Plasmodium falciparum dihydroorotate dehydrogenase (PfDHODH)
}

\author{
Zakari Ya'u Ibrahim ${ }^{*}$, Adamu Uzairu, Gideon Adamu Shallangwa and Stephen Eyije Abechi
}

\begin{abstract}
Background: The sixteen (16) designed data set of substituted aryl amine-based triazolopyrimidine were docked against Plasmodium falciparum dihydroorotate dehydrogenase (PfDHODH) employing Molegro Virtual Docker (MVD) software and their pharmacokinetic property determined through SwissADME predictor.

Results: The docking studies shows compound D16, 5-((6-methoxy-5-methyl-[1,2,4]triazolo[1,5-a]pyrimidin-7$\mathrm{yl}$ )amino)benzo[b]thiophen-4-ol to be the most interactive and stable derivative (re-rank score $=-114.205 \mathrm{kcal} /$ $\mathrm{mol}$ ) resulting from the hydrophobic as well as hydrogen interactions. The hydrogen interaction produced one hydrogen bond with the active residues LEU359 $(\mathrm{H} \cdot \mathrm{H} \cdot \mathrm{O} \cdot \mathrm{O})$ at a bond distances of $2.2874 \AA$. All the designed derivatives were found to pass the Lipinski rule of five tests, supporting the drug-likeliness of the designed compounds.

Conclusion: The ADME analysis revealed a perfect concurrence with the Lipinski Ro5, where the derivatives were found to possess good pharmacokinetic properties such as molar refractivity (MR), number of rotatable bonds (nRotb), log of skin permeability (log Kp), blood-brain barrier (BBB). These results could a deciding factor for the optimization of novel antimalarial compounds.
\end{abstract}

Keywords: Docking simulation, Pharmacokinetic, SwissADME prediction, Substituted aryl amine-based triazolopyrimidine, PfDHODH

\section{Background}

Malaria is an infectious disease spread by female Anopheles mosquitos that affect over half of the world's population [1] and kill over one million people each year. Human malaria is caused by the Plasmodium genus. Five Plasmodium species have been identified: P. ovale, $P$. vivax, P. falciparum, P. malariae, and P. knowlesi, with P. falciparum being the deadliest of all $[2,3]$. Because of their affordability and potency, antimalarial medications

\footnotetext{
* Correspondence: zakariyyadibrahim@gmail.com

Ahmadu Bello University, Zaria, Nigeria
}

like chloroquine, mefloquine, and antifolates have remained the standard treatment for the condition. Drug resistance to currently available antimalarial medications is a setback in the fight to eradicate the disease. As a result, researchers all over the world are motivated to develop new antimalarial medicines with diverse mechanisms of action [4].

Because the desired bases or nucleosides cannot be retrieved, the $P$. falciparum parasites can only obtain pyrimidine nucleotides through the de novo pyrimidine pathway [5]. As a result, finding novel targets in the pyrimidine biosynthesis pathway could be a promising
Springer Open (c) The Author(s). 2021 Open Access This article is licensed under a Creative Commons Attribution 4.0 International License, which permits use, sharing, adaptation, distribution and reproduction in any medium or format, as long as you give appropriate credit to the original author(s) and the source, provide a link to the Creative Commons licence, and indicate if changes were made. The images or other third party material in this article are included in the article's Creative Commons licence, unless indicated otherwise in a credit line to the material. If material is not included in the article's Creative Commons licence and your intended use is not permitted by statutory regulation or exceeds the permitted use, you will need to obtain permission directly from the copyright holder. To view a copy of this licence, visit http://creativecommons.org/licenses/by/4.0/. 
avenue for rare drug development [6]. Several compounds have been identified to target Plasmodium falciparum lactate dehydrogenase, PfLDH, and have been used as antimalarial medicines based on their activity with specific protein targets [7]; azaaurones have been shown to target the mitochondrial respiratory chain enzyme cytochrome bc1 [8], while $\beta$-carboline derivatives have been shown to target cytosolic malate dehydrogenase $(\mathrm{MDH})$, which helps transport metabolites to the mitochondria of $P$. falciparum [9]. The activity of arylamine-based triazolopyrimidine against $P$. falciparum has been observed $[10,11]$.

The PfDHODH enzyme can be present in both the cytoplasm and mitochondria of living organisms. The catalytic reaction of PfDHODH in the mitochondrial under the influence of Flavin mononucleotide (FMN) and coenzyme $\mathrm{Q}(\mathrm{CoQ})$ involves the oxidation of dihydroorotate into orotic acid [12], which is catalyzed by FMN, and the use of CoQ to re-oxidized the FMN. The $\beta / \alpha$-barrel fold catalytic domain of PfDHODH was generated by the amino-acid residues $162-56$. The residue to the domain $\mathrm{N}$-terminus anchors the protein present in the mitochondrial (inner membrane) [5]. The CoQ, which is situated between the domains of the $\beta / \alpha$-barrel and the $\mathrm{N}$-terminal $\alpha$-helical membrane, is a binding site for several DHODH inhibitors. The speciesselectivity of these inhibitors is due to a difference in amino acid sequence between the PfDHODH and hDHODH enzymes $[13,14]$.

A plethora of studies on the molecular docking and pharmacokinetic properties of antimalarial compounds have been published, including one on the docking and ADME determination of -Amino alcohol grafted 1,4,5-trisubstituted 1,2,3-triazoles derivatives to elevating p53 protein [15]; in 2020, Gorki and his colleagues conducted docking analysis and evaluation of $\beta$-carboline derivatives [9], Dohutia and his colleagues in 2018 on novel curcumin analogs [16], and Prakash and Co. in 2010 on antimalarial docking studies [17]. The study focuses on the prediction of pharmacokinetic properties of sixteen substituted aryl aminebased triazolopyrimidine derivatives and their molecular docking as PfDHODH inhibitors to account for the causes of their interactions using the Molegro Virtual Docker (MVD).

\section{Methods}

\section{Software and materials}

The docking simulations were carried out with DELL Inspiron personal computer with the following specifications: COREi7 processor, 8GB RAM, 1 TB graphics card, and 1000 GB hard disc capacity. The docking studies were performed with Molegro Virtual Docker (MVD) software while the SwissADME software was employed in predicting the pharmacokinetic properties of the compounds.

\section{Preparation of ligands}

The 2-dimensional structures of the sixteen (16) designed derivatives of substituted aryl amine-based triazolopyrimidine [18] presented in Table 1 were sketched with ChemDraw Ultra 12.0. These structures were then opened in Spartan'14 version 1.1.2 software in a 3dimensional format and are optimized on density functional theory, DFT/B3LYP, and a basis set of $6-31+\mathrm{G}^{*}$. The optimized geometries were thereafter saved in $\mathrm{pdb}$ file format to be opened in the MVD for docking.

\section{Preparation of protein}

The 3D structure of Plasmodium falciparum dihydroorotate dehydrogenase (PfDHODH) [PDB ID: 4OQV] with resolution $1.23 \AA$ was extracted from the protein data bank and saved in pdb file format. The protein was prepared by replacing any missing hydrogen using the MVD protein reparation wizard. The cavity detection wizard locates the binding spot of the protein for the protein action.

\section{Docking parameters}

The docking parameters selected for the analysis include picking PlantScore Grid as the scoring function, over a 0.3- $\AA$ grid resolution. The docking radius was then fixed at 18 covering over $90 \%$ of the protein cavities detected. The MolDock SE searching algorithm was selected in addition to checking the energy minimization, constrain poses to the cavity, and optimize H-bonds boxes. The iterations were set at 1500 maximum, population size set at 50 maximum, and energy threshold equal to 100.00 leaving the Tries values for min, quick and max tries equals to 10,10 , and 30 , respectively. The default respectively values of 300 and 1.00 for the max step and neighbor distance factor. Furthermore, the energy threshold was enabled in the pose clustering dialog box.

\section{Molecular docking}

The orientation and the molecular interactions between the designed derivatives of substituted aryl amine-based triazolopyrimidine with their protein target are established by molecular docking studies. The docking studies were carried out with the aid of the Molegro Virtual Docker (MVD) software on the PfDHODH. The cavity detection wizard locates the binding spots before the designed derivatives were imported for the studies of molecular interactions. The ribbon diagram of Plasmodium falciparum dihydroorotate dehydrogenase (PfDHODH) with the designed derivatives of substituted aryl aminebased triazolopyrimidine are indicated in Fig. 1. 
Table 1 IUPAC Names, structures, as well as the predicted activities of the designed derivatives of substituted aryl aminebased triazolopyrimidine inhibitors of PfDHODH

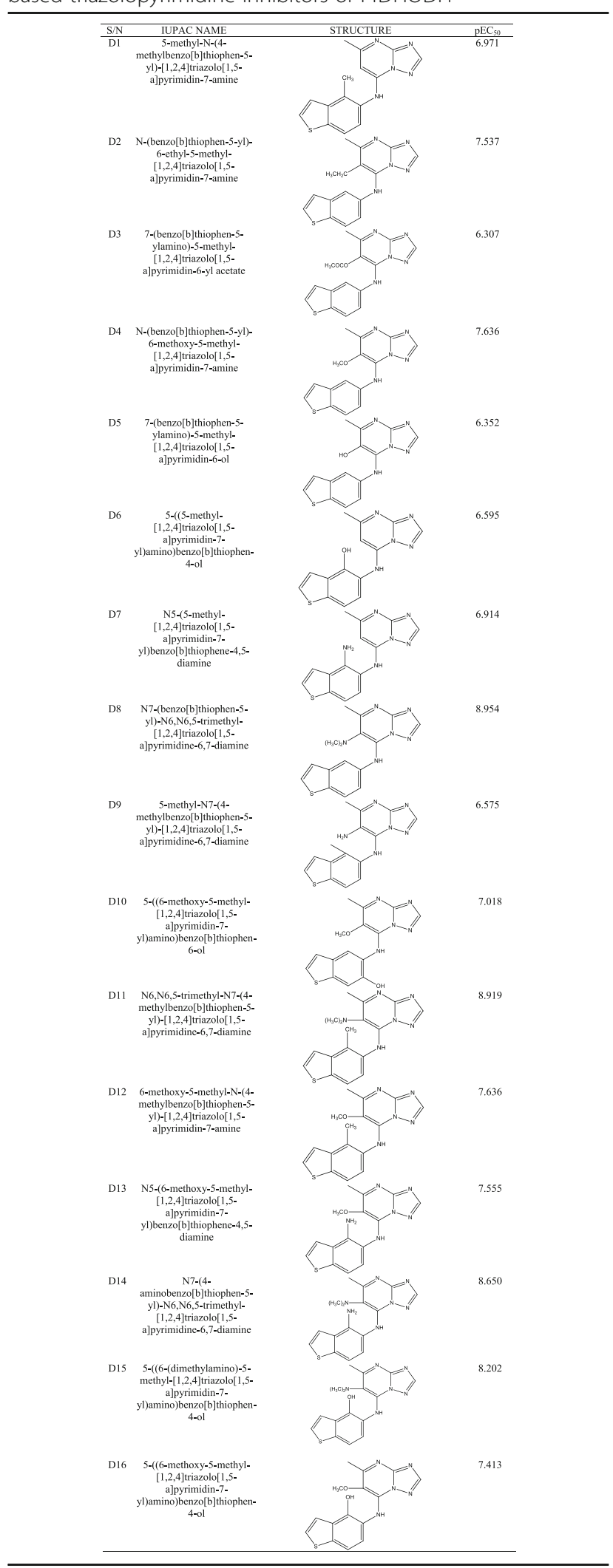

\section{Drug-likeness and ADME prediction}

The SwissADME software (http://www.swissadme.ch) was an online tool for determining the drug-likeness and pharmacokinetic parameters of the proposed derivatives. Using Lipinski's rule of 5, the drug-likeness of the compounds was predicted. The guideline was created to establish ground rules for new molecular entities in terms of drug-likeness [19]. According to the rule of 5, molecules having $\mathrm{H}$-bond donors greater than 5 , $\mathrm{H}$-bond acceptors greater than 10 , a molecular weight larger than 500, and $\log \mathrm{P}(\mathrm{iLog} \mathrm{P})$ larger than 5. Further parameters like topological polar surface area (TPSA) $<140 \AA^{2}$ and number of rotatable bonds (nRotb) were reported [20] to have poor absorption. The pharmacokinetic properties to be determined include the molar refractivity (MR), log of skin permeability (log Kp), blood-brain barrier (BBB) penetration, permeability glycoprotein (Pgp) substrate, gastrointestinal (GI) absorption, and cytochrome P450 (CYP450) enzymes: CYP1A2, CYP2C9, and CYP2C19 inhibitors.

\section{Results \\ Docking studies}

The docking results, numbers of H-bond(s) with their energies, and the interaction energies are shown in Table 2 while Table 3 provides the details of the hydrogen bonding of the most active derivatives along with their distance. The docking pose of the D2 derivative (most stable) is reflected in Fig. 2.

\section{Drug-likeness and ADME prediction}

The results of the analysis on Lipinski's parameters of the designed derivatives are reflected in Table 4 while Table 5 shows the results of the pharmacokinetic properties of the designed derivatives.

\section{Discussion}

Docking studies

Table 2 shows the docking scores of all the designed compounds against the protein receptor, which were compared to the chloroquine standard. The number of hydrogen bonds, hydrogen bond energy, and interaction energy were all included in the table (Table 2). All the compounds (except D1, - 98.7673; D3, - 97.6691; D6, 98.84; D13, - 101.897; and D15, - $96.0251 \mathrm{kcal} / \mathrm{mol}$ ) were found to have a higher docking score than the chloroquine standard $(-102.393 \mathrm{kcal} / \mathrm{mol})$. As a result, they have a higher binding affinity than the normal chloroquine. The higher interaction energies (Table 2) of compounds D2, D7, D9, D10, D11, D14, and D16 with energies of - 155.075, - 147.869, - 142.607, 155.332, - 143.971, - 143.887, and $-141.429 \mathrm{kcal} / \mathrm{mol}$, respectively, confirmed their better interaction with the target than the chloroquine standard $(-139.888 \mathrm{kcal} /$ 


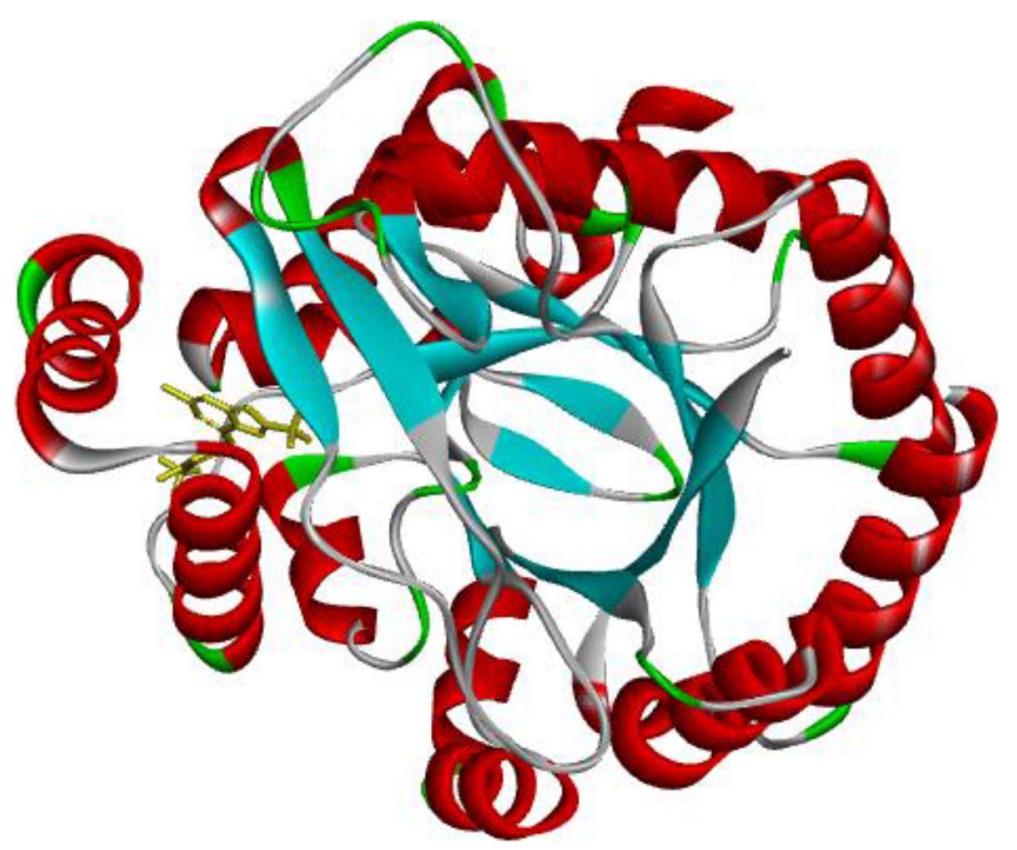

Fig. 1 Ribbon diagram showing the derivatives of substituted aryl amine-based triazolopyrimidine (yellow ribbon) in the binding site of $\mathrm{PfDHODH}$ (reddish ribbon with blue and white stripes)

mol). As demonstrated in Table 3, the proposed ligands generated hydrogen bonds with active site residues such as LEU359, TYR356, PRO52, HIS56, ARG136, and TYR147. The hydrogen bonds for six of the most active ligands are detailed in Table 3. These compounds are D16, 5-((6-methoxy-5-methyl-[1,2,4]triazolo[1,5-a]pyrimidin-7-yl)amino)benzo[b]thiophen-4-ol; $\quad$ D2, N- (benzo[b]thiophen-5-yl)-6-ethyl-5-methyl-[1,2,4] triazolo [1,5-a]pyrimidin-7-amine; D10, 5-((6-methoxy-5-methyl$[1,2,4]$ triazolo[1,5-a]pyrimidin-7-yl)amino)benzo[b]thiophen-6-ol; D11, N6, N6,5-trimethyl-N7-(4-methylbenzo[b]thiophen-5-yl)-[1,2,4] triazolo[1,5-a]pyrimidine-6,7diamine; D7, N5-(5-methyl-[1,2,4]triazolo[1,5-a]pyrimidin-7-yl)benzo[b]thiophene-4,5-diamine; and D9, 5-

Table 2 The docking studies results, no. of $\mathrm{H}$-bond(s), H-bond, and interaction energies

\begin{tabular}{llllll}
\hline S/N & MolDock score $(\mathbf{k c a l} / \mathbf{m o l})$ & Re-rank score $\mathbf{( k c a l / m o l )}$ & No. of H-bond(s) & H-bond $(\mathbf{k c a l} / \mathbf{m o l})$ & Interaction $(\mathbf{k c a l} / \mathbf{m o l})$ \\
\hline D1 & -125.748 & -106.396 & 2 & -4.959 & -131.256 \\
D2 & -152.985 & -123.259 & 2 & -7.063 & -155.075 \\
D3 & -123.482 & -97.669 & 2 & -8.243 & -124.417 \\
D4 & -134.62 & -107.683 & 1 & -4.212 & -138.535 \\
D5 & -123.977 & -106.921 & 1 & -1.693 & -132.195 \\
D6 & -117.671 & -98.840 & 1 & -1.190 & -126.461 \\
D7 & -142.282 & -119.256 & 3 & -7.342 & -147.869 \\
D8 & -134.134 & -108.239 & 1 & -4.071 & -138.861 \\
D9 & -138.674 & -116.174 & 1 & -4.994 & -142.607 \\
D10 & -146.64 & -121.678 & 2 & -10.972 & -155.332 \\
D11 & -139.674 & -119.665 & 1 & -4.909 & -150.307 \\
D12 & -132.062 & -109.956 & 1 & -1.570 & -138.590 \\
D13 & -129.247 & -101.897 & 1 & -4.672 & -137.136 \\
D14 & -143.168 & -111.957 & 2 & -4.607 & -143.887 \\
D15 & -111.061 & -96.025 & 2 & -5.550 & -121.342 \\
D16 & -135.509 & -114.205 & 1 & -3.657 & -141.429 \\
Chloroquine & -126.017 & -102.393 & 3 & -5.936 & -139.888 \\
\hline
\end{tabular}


Table 3 Hydrogen bonding details between the protein receptor and six of the most active derivatives

\begin{tabular}{|c|c|c|c|c|c|c|c|}
\hline \multirow[b]{2}{*}{ Name } & \multirow[b]{2}{*}{ H-bond(s) } & \multicolumn{2}{|c|}{ H-binding ligand } & \multirow[b]{2}{*}{ Residue } & \multicolumn{2}{|c|}{ H-binding receptor } & \multirow[b]{2}{*}{ H-bond distance $(\AA ̊)$} \\
\hline & & Element & Type & & Element & Type & \\
\hline D16 & 1 & $\mathrm{H}$ & $\mathrm{D}$ & Leu359 & $\mathrm{O}$ & A & 2.2874 \\
\hline \multirow[t]{2}{*}{ D2 } & 2 & N & A & Tyr356 & $\mathrm{H}$ & D & 2.1268 \\
\hline & & $\mathrm{H}$ & $\mathrm{D}$ & Pro52 & $\mathrm{O}$ & A & 2.0042 \\
\hline \multirow[t]{2}{*}{ D10 } & 2 & $\mathrm{O}$ & A & His56 & $\mathrm{H}$ & $\mathrm{D}$ & 2.1519 \\
\hline & & N & A & $\operatorname{Arg} 136$ & $\mathrm{H}$ & $\mathrm{D}$ & 2.2784 \\
\hline D11 & 1 & $\mathrm{~N}$ & A & $\operatorname{Arg} 136$ & $\mathrm{H}$ & $\mathrm{D}$ & 1.6277 \\
\hline \multirow[t]{3}{*}{ D7 } & 3 & N & A & Tyr356 & $\mathrm{H}$ & $\mathrm{D}$ & 2.1607 \\
\hline & & $\mathrm{H}$ & D & Tyr147 & $\mathrm{O}$ & A & 2.1484 \\
\hline & & $\mathrm{H}$ & $\mathrm{D}$ & Pro52 & $\mathrm{O}$ & A & 2.3053 \\
\hline D9 & 1 & $\mathrm{~N}$ & A & Arg136 & $\mathrm{H}$ & $D$ & 1.6080 \\
\hline
\end{tabular}

methyl-N7-(4-methylbenzo[b]thiophen-5-yl)-[1,2,4]triazolo[1,5-a]pyrimidine-6,7-diamine, with - 114.205, 123.259, - 121.678, - 119.665, - 119.256, and - 116.174 $\mathrm{kcal} / \mathrm{mol}$ re-rank scores, respectively. A hydrogen bond is formed between compound D16 and amino acid residue, LEU359 (H...H..O) with a bond distance of $2.2874 \AA$. The two hydrogen bonds formed between compound D2 and the active residues TYR356 ( $\mathrm{N} \cdot \cdot \mathrm{H} \cdot \cdot \mathrm{H})$ and PRO52 ( $\mathrm{H} . \mathrm{H} \cdot . \mathrm{O})$ have bond distances 2.12677 and $2.00418 \AA$, respectively, while those between D10 with the active residue HIS56 $(\mathrm{O} \cdot \cdot \mathrm{H} \cdot \cdot \mathrm{H})$ and ARG136 $(\mathrm{N} . . \mathrm{H} \cdot . \mathrm{H})$ have bond distances 2.15191 and $2.27840 \AA$, respectively. The interactions of D11 with ARG136 $(\mathrm{N} \cdot . \mathrm{H} \cdot \mathrm{H})$ residue are at a $1.6277-\AA$ bond distance. The three hydrogen bond interactions of D7 with TYR356 $(\mathrm{N} \cdot \cdot \mathrm{H} \cdot \cdot \mathrm{H}), \quad$ TYR145 $(\mathrm{H} \cdot . \mathrm{H} \cdot . \mathrm{O})$, and PRO52 $(\mathrm{H} \cdot \cdot \mathrm{H} \cdot . \mathrm{O})$ amino acid residues gave rise to $2.1607,2.1484$, and $2.3053 \AA$ bond distances, respectively. The lone hydrogen bond formed between D9 and ARG136 (N..H..H) residue is at a distance of $1.6080 \AA$. The presence of hydrogen bonds, in addition to other hydrophobic interactions, may explain the high docking scores of the most active molecules. The binding modes as well as interaction images of the most active compounds, D16, D2, D10, D11, and D7, were exhibited in Fig. 2.

\section{Drug-likeness and ADME prediction}

The Lipinski, rule-of-five (Ro5), is used to assess the drug-likeness of chemical compounds and potential medicines. According to Lipinski's Ro5, chemical compounds that can be utilized as pharmaceuticals should have a molecular weight (MW) of less than $500 \mathrm{~g} / \mathrm{mol}$, a logarithm of the partition coefficient $(\log \mathrm{P})$ of less than 5 , hydrogen bond donors (HBDs) of less than 5, and a hydrogen bond acceptor (HBA) of less than 10 [21]. Furthermore, the number of rotatable bonds (RotB) of $\leq 10$ and a topological polar surface area (TPSA) of $\leq 140 \AA^{2}$ $[20,22-24]$ have been observed to correlate with pharmacological flexibility and permeability, respectively. Compounds that meet these criteria have been shown to have better pharmacokinetics and bioavailability characteristics.

Low molecular weight (MW) signifies that the molecules are light and can easily pass through the cell membrane. Low molecular weight (MW 500) chemicals are favored for oral absorption [25], whereas compounds with $\mathrm{MW}>500 \mathrm{Da}$ are absorbed via an alternate route, generally the membrane [26]. The research revealed that all of the data (Table 4) were less than $500 \mathrm{Da}$.

The implicit $\log \mathrm{P}(\mathrm{I} \log \mathrm{P})$ is the $\mathrm{n}$-octanol/water partition coefficients of a particular molecule in two immiscible solvents; it dissolves the molecule in both solvents while maintaining the molecule's neutrality. Initially, the IlogP was hired for biomedical and pharmaceutical research. IlogP plays a critical role in medication absorption in the mouth [25], as well as facilitating drug interactions with their biological targets [27]. Because it possesses both hydrophilic and lipophilic qualities, noctanol was thought to be a superb mimic of phospholipid membrane features [28]. The estimated values of IlogP (Table 4) were found to be less than five (1.823.05), as recommended by Lipinski's rule of five [25]. As a result, the developed derivatives should have great oral absorption qualities.

H-bond acceptors (HBA) number is as follows: Any heteroatom with at least one bound hydrogen is referred to as a hydrogen bond acceptor. The sum of these heteroatoms ( $\mathrm{N}$ and $\mathrm{O}$ atoms) should be fewer than 10 according to the Lipinski rule of five [25]. The H-bond acceptors determined for the intended compounds (Table 4) ranged from 3 to 5 , which is significantly less than the Ro5 projected maximum limit.

$\mathrm{H}$-bond donor (HBD) count is as follows: Any heteroatom lacking a formal positive charge, save pyrrole nitrogen, halogens, sulfur, heteroaromatic oxygen, and higher oxidation states of nitrogen, phosphorus, and sulfur, but 


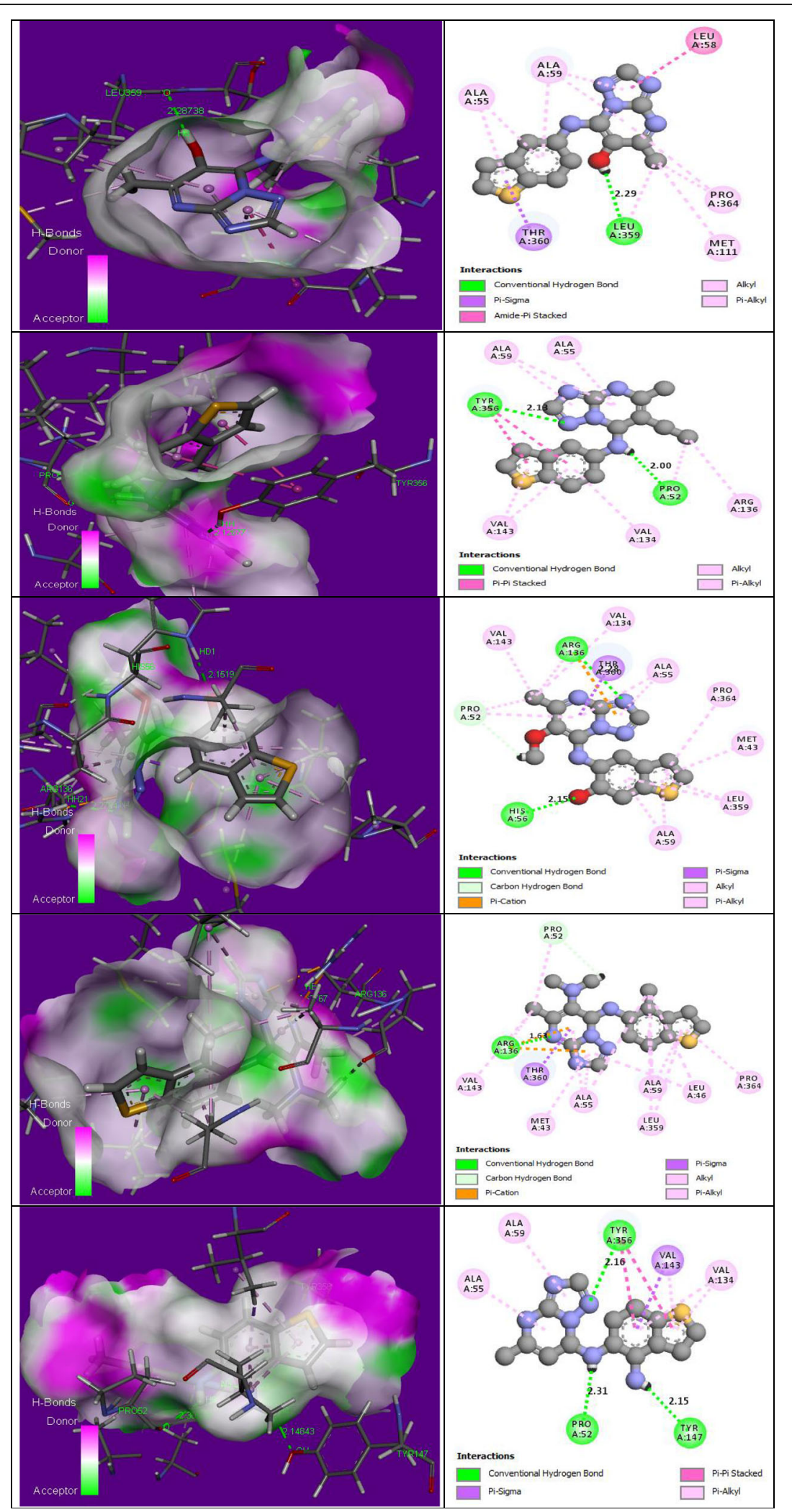

Fig. 2 Compounds D16, D2, D10, D11, and D7 respectively showing the binding modes as well as their interaction images with the protein 
Table 4 Lipinski's and Veber parameters of the designed derivatives of substituted aryl amine-based triazolopyrimidine

\begin{tabular}{|c|c|c|c|c|c|c|c|}
\hline \multirow[b]{2}{*}{$\mathrm{S} / \mathrm{N}$} & \multicolumn{4}{|c|}{ Lipinski's parameters } & \multirow[b]{2}{*}{ Lipinski \#violations } & \multicolumn{2}{|l|}{ Veber parameters } \\
\hline & $\mathrm{MW}(\leq 500 \mathrm{Da})$ & $\operatorname{logp}(<5)$ & $\#$ H-bond acceptors $(\leq 10)$ & \#H-bond donors $(\leq 5)$ & & TPSA $\left(<140 \AA^{2}\right)$ & nRotb \\
\hline D1 & 295.36 & 2.86 & 3 & 1 & 0 & 83.35 & 2 \\
\hline D2 & 309.39 & 2.72 & 3 & 1 & 0 & 83.35 & 3 \\
\hline D3 & 339.37 & 2.72 & 5 & 1 & 0 & 109.65 & 4 \\
\hline D4 & 311.36 & 2.72 & 4 & 1 & 0 & 92.58 & 3 \\
\hline D5 & 297.34 & 1.82 & 4 & 2 & 0 & 103.58 & 2 \\
\hline D6 & 297.34 & 2.38 & 4 & 2 & 0 & 103.58 & 2 \\
\hline D7 & 296.35 & 2.31 & 3 & 2 & 0 & 109.37 & 2 \\
\hline D8 & 324.40 & 2.87 & 3 & 1 & 0 & 86.59 & 3 \\
\hline D9 & 310.38 & 2.16 & 3 & 2 & 0 & 109.37 & 2 \\
\hline D10 & 327.36 & 2.51 & 5 & 2 & 0 & 112.81 & 3 \\
\hline D11 & 338.43 & 3.05 & 3 & 1 & 0 & 86.59 & 3 \\
\hline D12 & 325.39 & 2.93 & 4 & 1 & 0 & 92.58 & 3 \\
\hline D13 & 326.38 & 2.50 & 4 & 2 & 0 & 118.60 & 3 \\
\hline D14 & 339.42 & 2.40 & 3 & 2 & 0 & 112.61 & 3 \\
\hline D15 & 340.40 & 2.64 & 4 & 2 & 0 & 106.82 & 3 \\
\hline D16 & 327.36 & 2.41 & 5 & 2 & 0 & 112.81 & 3 \\
\hline
\end{tabular}

Molecular weight (MW), log of octanol/water partition coefficient (ilogP), hydrogen bond acceptor(s) (\#H-bond acceptors), hydrogen bond donor(s) (\#H-bond donors), and topological polar surface area (TPSA), Molecular weight (MW), Log of octanol/water partition coefficient (ilogP), hydrogen bond acceptor(s) (\#H-bond acceptors), hydrogen bond donor(s) (\#H-bond donors), topological polar surface area (TPSA), and number of rotatable bonds (nRotb)

Table 5 Pharmacokinetics properties of the designed derivatives of substituted aryl amine-based triazolopyrimidine

\begin{tabular}{|c|c|c|c|c|c|c|c|c|}
\hline $\mathrm{S} / \mathrm{N}$ & MR & $\log K p(\mathrm{~cm} / \mathrm{s})$ & Gl absorption & BBB permeant & P-gp substrate & CYP1A2 inhibitor & CYP2C19 inhibitor & CYP2C9 inhibitor \\
\hline D1 & 85.64 & -5.43 & High & No & No & Yes & Yes & Yes \\
\hline D2 & 90.45 & -5.21 & High & No & No & Yes & Yes & Yes \\
\hline D3 & 92.17 & -6.14 & High & No & No & Yes & Yes & Yes \\
\hline D4 & 87.17 & -5.81 & High & No & No & Yes & Yes & Yes \\
\hline D5 & 82.70 & -5.96 & High & No & No & Yes & Yes & No \\
\hline D6 & 82.70 & -5.96 & High & No & No & Yes & Yes & No \\
\hline D7 & 85.08 & -6.18 & High & No & Yes & Yes & No & No \\
\hline D8 & 94.88 & -5.79 & High & No & No & Yes & Yes & Yes \\
\hline D9 & 90.05 & -6.01 & High & No & Yes & Yes & Yes & Yes \\
\hline D10 & 89.19 & -6.16 & High & No & No & Yes & No & Yes \\
\hline D11 & 99.85 & -5.61 & High & No & No & Yes & Yes & Yes \\
\hline D12 & 92.13 & -5.64 & High & No & No & Yes & Yes & Yes \\
\hline D13 & 91.57 & -6.39 & High & No & Yes & Yes & No & Yes \\
\hline D14 & 99.29 & -6.36 & High & No & Yes & Yes & No & Yes \\
\hline D15 & 96.90 & -6.13 & High & No & No & Yes & No & Yes \\
\hline D16 & 89.19 & -6.16 & High & No & No & Yes & No & Yes \\
\hline
\end{tabular}

Molar refractivity (MR), log of skin permeability (log Kp), blood-brain barrier (BBB) penetration, permeability glycoprotein (Pgp) substrate, gastrointestinal (GI) absorption, cytochrome P450 (CYP450) enzymes: CYP1A2, CYP2C9, and CYP2C19 inhibitors 
including the oxygens connected to them, is referred to as a hydrogen bond donor. The amount of hydrogen bond donors (the sum of the $\mathrm{OH}$ and $\mathrm{NH}$ groups) should be less than or equal to 5 according to the Ro5. As can be seen in Table 4, all of the HBD values obtained were less than 5 . Both HBA and HBD were critical because they synergize between chemicals and macromolecules, as well as having the potential to determine oral absorption [25].

The TPSA of a molecule is the sum of all polar atoms (oxygen, nitrogen, and their connected hydrogens) on the molecule's surface, calculated by adding all polar fragments [29]. The goal of the TPSA is to predict drug transport qualities such as intestinal absorption [30] and $\mathrm{BBB}$ penetration [31]. For virtual screening and ADME property prediction, TPSA has gained prominence in medicinal chemistry [32]. When the quantitative value of TPSA is $<140 \AA^{2}$, it becomes a good predictor of intestinal absorption, and when it is $<60 \AA^{2}$, it indicates good blood-brain barrier penetration [33]. The proposed derivatives' TPSA values (Table 4) were found to range from 83.35 to $118.60 \AA^{2}$. This indicates that the results are less than $140 \AA^{2}$, indicating that intestinal absorption is good. However, because the TPSA values are larger than $60 \AA^{2}$, the proposed derivatives do not penetrate the blood-brain barrier well, as evidenced by the BBB determination (Table 5).

The total number of rotatable bonds (RBN) is equal to the total number of bonds that may freely spin around themselves. They are non-ring single bonds with a nonterminal heavy atom attached (i.e., non-hydrogen). Molecules with less than ten rotatable bonds have been reported to have better oral availability [20]. The number of rotatable bonds for the developed compounds was determined to be less than 5 , indicating that the developed compounds had a good oral bioavailability.

The in silico ADME studies involve investigating some pharmacokinetic properties of the designed compounds such as molar refractivity (MR), log of skin permeability (log Kp), blood-brain barrier (BBB) penetration, permeability glycoprotein (Pgp) substrate, gastrointestinal (GI) absorption, and cytochrome P450 (CYP450) enzymes: CYP1A2, CYP2C9, and CYP2C19 inhibitors. The reciprocal of the volume of a mole of a substance is defined as the molar refractivity (MR). The overall polarizability of a mole of a substance is related to molar refractivity. Molar refractivity data provide information about the electronic polarizability of individual ions in solution [34]. The refractive index results can be used to explain molecular interactions in solution [35]. The molar refractivity value should be between 40 and 130 for good absorption and oral bioavailability. Acceptable molar refractivity values, in combination with the number of rotatable bonds, indicate that substances have adequate intestinal absorption and oral bioavailability [15]. The designed compound's MR values range from 82.7 to $99.85 \mathrm{~m} 3 / \mathrm{mol}$. This indicates that the proposed compounds have good intestinal absorption and oral bioavailability.

Permeability is a critical component of drug research since it predicts metabolite absorption, distribution, metabolism, and excretion (ADME). The ability of molecules to penetrate the outer layer of the skin is described by skin permeability (Kp) [36]. The Kp includes assessing a compound's biological absorption via the skin and has been used as a source of data for threat assessment on the skin [37]. The developed compounds' log Kp values (Table 5) were all determined to be within the permissible range of -8.0 to -1.0 [38].

The blood-brain barrier is a microvascular endothelial layer of cells that surrounds the central nervous system (CNS) (BBB). The BBB is a structural and chemical barrier that prevents various medications from entering the brain, making the use of newly produced medications in the treatment of brain illnesses or other brain-related issues ineffective. Several prospective therapeutic compounds have been discovered to provide a significant obstacle to therapeutic research for central nervous system illnesses if they have minimal or no BBB penetration. The results of the $\mathrm{BBB}$ permeability test performed on our proposed derivatives (Table 5) demonstrated that all of them lack BBB permeability, making their application in the treatment of cerebral malaria futile.

The adenosine triphosphate (ATP)-binding cassettetransporter permeability glycoprotein (Pgp) functions primarily as a carrier-mediated primary active efflux transporter. P-glycoprotein can bind to a wide variety of substrates, which are widely distributed throughout the body. Pgp transporters are located in the small intestine, blood-brain barrier capillaries, and several critical organs such as the kidney and liver [39]. Substances can enter the cell via active transport or passive diffusion, and they can be effluxed with the help of Pgp. The Pgp affects the absorption, distribution, and clearance of a variety of substances. As a result, identifying permeability glycoprotein substrates is critical for identifying prospective medicines and optimizing them. Pgp substrate was detected only in the proposed compounds D7, D9, D13, and D14.

Cytochrome P450 (CYP) enzymes are a family of proteins involved in the synthesis and metabolism of a wide range of internal and exterior cellular components. These enzymes have been found in animals, plants, microorganisms, and even a few viruses. They get their name from the fact that they are linked to the cell membrane (cyto) and contain heme pigment (chrome and P), which produces a $450 \mathrm{~nm}$ spectrum when combined with carbon monoxide. 
In humans, heme-containing cytochromes P450 (CYPs) are a superfamily of enzymes that break down a variety of endogenous and xenobiotic substances. More than 50 isoforms of CYP enzymes exist, with 1A2, 2C9, 2C19, 2D6, and 3A4 isoforms accounting for over $90 \%$ of oxidative metabolic processes [36]. Inhibitory drug metabolism fails when CYP enzymes are inhibited. During medication development, studying the inhibitory activity of proposed derivatives against a certain CYP isoform becomes a critical factor. Table 5 shows the results of the inhibitory prediction for three CYP isoforms (CYP1A2, CYP2C9, and CYP2C19). While all of the proposed compounds were anticipated to inhibit CYP1A2, just a few derivatives (D7, D10, and D13-16) were found not to inhibit CYP2C19, and just three derivatives (D57) were found to inhibit CYP2C9.

\section{Conclusions}

SwissADME and Molegro Virtual Docker were used to determine the pharmacokinetics and docking investigations of the sixteen (16) substituted aryl amine-based triazolopyrimidine derivatives. Because none of the substances violated Lipinski's rule of five, their pharmacokinetic characteristics are sound. When compared to the common drug chloroquine, the binding affinities they demonstrated are promising, indicating a stronger binding interaction with the target protein, PfDHODH. Due to its lowest docking score (re-rank score $=-114.205$ $\mathrm{Kcal} / \mathrm{mol})$, molecule D16, 5-((6-methoxy-5-methyl-[1,2, 4] triazolo[1,5-a]pyrimidin-7-yl)amino)benzo[b]thiophen4-ol, was found to be the most stable of all the derivatives. The activity of the compound could be attributed to the hydrogen bond present in the molecule, as well as other hydrophobic interactions. Because of their superior pharmacokinetic properties, the derivatives could be used to treat malaria.

\section{Abbreviations \\ PfLDH: Plasmodium falciparum lactate dehydrogenase; MDH: Malate dehydrogenase; PfDHODH: Plasmodium falciparum dihydroorotate dehydrogenase; MVD: Molegro Virtual Docker; hDHODH: Human dihydroorotate dehydrogenase; MR: Molar refractivity; nRotb: Number of rotatable bonds; log Kp: Log of skin permeability; BBB: Blood-brain barrier; Pgp: Permeability glycoprotein substrate; GI: Gastrointestinal absorption; Cytochrome P450 (CYP450) enzymes: CYP1A2, CYP2C9, and CYP2C19 inhibitors; MW: Molecular weight; ilogP: Log of octanol/water partition coefficient; TPSA: Total polar surface area}

\section{Acknowledgements}

We are thankful to the members of staff in the physical chemistry unit, chemistry department of Ahmadu Bello University, for providing the essential facilities to carry out this research work.

\section{Authors' contributions}

This research involves the combined efforts of all the authors: $\mathrm{ZYI}$ and $\mathrm{AU}$ conceived and designed the research; ZYI and GAS performed the theoretical studies; ZYI, AU, and SEA analyzed and interpreted the data; ZYI, GAS, and SEA contributed materials and data set; and ZYI and SEA wrote the manuscript. All authors read and approved the final manuscript.

\section{Funding}

The authors of this research did not receive any funding concerning this research.

\section{Availability of data and materials}

The datasets used for analysis during these studies were included in this published study.

\section{Declarations}

Ethics approval and consent to participate

Not applicable.

Consent for publication

Not applicable.

\section{Competing interests}

The authors declare that they have no competing interests.

Received: 15 February 2021 Accepted: 19 June 2021

Published online: 05 July 2021

\section{References}

1. WHO (2019) World malaria report 2019. World Health Organization, Geneva

2. Kwenti TE, Kwenti TDB, Njunda LA, Latz A, Tufon KA, Nkuo-Akenji T (2017) Identification of the Plasmodium species in clinical samples from children residing in five epidemiological strata of malaria in Cameroon. Trop Med Health 45(1):14. https://doi.org/10.1186/s41182-017-0058-5

3. Bhat HR, Ghosh SK, Prakash A, Gogoi K, Singh UP (2012) In vitro antimalarial activity and molecular docking analysis of 4-aminoquinoline-clubbed 1,3,5triazine derivatives. Lett Appl Microbiol 54(5):483-486. https://doi.org/1 0.1111/j.1472-765x.2012.03234.x

4. Borstnik K, Paik IH, Posner GH (2002) Malaria: new chemotherapeutic peroxide drugs. Mini Rev Med Chem 2(6):573-583. https://doi.org/10.21 74/1389557023405620

5. Phillips AM, Rathod KP (2010) Plasmodium dihydroorotate dehydrogenase: a promising target for novel anti-malarial chemotherapy. Infect Disord Drug Targets 10(3):226-239

6. Cassera MB, Zhang Y, Hazleton KZ, Schramm VL (2011) Purine and pyrimidine pathways as a target in Plasmodium falciparum. Curr Top Med Chem 11(16):2103-2115. https://doi.org/10.2174/156802611796575948

7. Tahghighi A, Mohamadi-Zarch S-M, Rahimi H, Marashiyan M, Maleki-Ravasan $\mathrm{N}$, Eslamifar A (2020) In silico and in vivo anti-malarial investigation on 1(heteroaryl)-2-((5-nitroheteroaryl)methylene) hydrazine derivatives. Malar J 19(1):231. https://doi.org/10.1186/s12936-020-03269-7

8. Hadni H, Elhallaoui M (2020) 2D and 3D-QSAR, molecular docking and ADMET properties in silico studies of azaaurones as antimalarial agents. New J Chem 44(16):6553-6565. https://doi.org/10.1039/c9nj05767f

9. Gorki V, Walter NS, Singh R, Chauhan M, Dhingra N, Salunke DB, Kaur S (2020) $\beta$-carboline derivatives tackling malaria: biological evaluation and docking analysis. ACS Omega 5(29):17993-18006. https://doi.org/10.1021/a csomega.0c01256

10. Gujjar R, Marwaha A, El Mazouni F, White J, White KL, Creason S et al (2009) Identification of a metabolically stable triazolopyrimidine-based dihydroorotate dehydrogenase inhibitor with antimalarial activity in mice. J Med Chem 52(7):1864-1872. https://doi.org/10.1021/jm801343r

11. Gujjar R, El Mazouni F, White KL, White J, Creason S, Shackleford DM et al (2011) Lead optimization of aryl and aralkyl amine-based triazolopyrimidine inhibitors of Plasmodium falciparum dihydroorotate dehydrogenase with antimalarial activity in mice. J Med Chem 54(11):3935-3949. https://doi. org/10.1021/jm200265b

12. Pavadai E, El Mazouni F, Wittlin S, de Kock C, Phillips MA, Chibale K (2016) Identification of new human malaria parasite Plasmodium falciparum dihydroorotate dehydrogenase inhibitors by pharmacophore and structurebased virtual screening. J Chem Inf Model 56(3):548-562. https://doi.org/1 0.1021 /acs.jcim.5b00680

13. Deng X, Gujiar R, El Mazouni F, Kaminsky W, Malmquist NA, Goldsmith EJ et al (2009) Structural plasticity of malaria dihydroorotate dehydrogenase allows selective binding of diverse chemical scaffolds. J Biol Chem 284(39): 26999-27009. https://doi.org/10.1074/jbc.m109.028589 
14. Deng X, Kokkonda S, El Mazouni F, White J, Burrows JN, Kaminsky W, Charman SA, Matthews D, Rathod PK, Phillips MA (2014) Fluorine modulates species selectivity in the triazolopyrimidine class of Plasmodium falciparum dihydroorotate dehydrogenase inhibitors. J Med Chem 57(12):5381-5394. https://doi.org/10.1021/jm500481t

15. Ibrahim ZY, Uzairu A, Shallangwa G, Abechi S (2020) Molecular docking studies, drug-likeness and in-silico ADMET prediction of some novel $\beta$ amino alcohol grafted 1,4,5-trisubstituted 1,2,3-triazoles derivatives as elevators of p53 protein levels. Sci Afr:e00570. https://doi.org/10.1016/j.scia f.2020.e00570

16. Dohutia C, Chetia D, Gogoi K, Bhattacharyya DR, Sarma K (2018) Molecular docking, synthesis and in vitro antimalarial evaluation of certain novel curcumin analogues. Braz J Pharm Sci 53(4). https://doi.org/10.1590/s217597902017000400084

17. Prakash N, Patel S, Faldu JN, Ranjan R, Sudheer DVN (2010) Molecular docking studies of antimalarial drugs for malaria. J Comput Sci Syst Biol 03(03). https://doi.org/10.4172/jcsb.1000059

18. Ibrahim ZY, Uzairu A, Shallangwa G, Abechi S (2020) In-silico design of aryl and aralkyl amine-based triazolopyrimidine derivatives with enhanced activity against resistant Plasmodium falciparum. Chem Afr 4(1):137-148. https://doi.org/10.1007/s42250-020-00199-4

19. Lipinski CA (2000) Drug-like properties and the causes of poor solubility and poor permeability. J Pharmacol Toxicol Methods 44(1):235-249. https://doi. org/10.1016/s1056-8719(00)00107-6

20. Veber DF, Johnson SR, Cheng H-Y, Smith BR, Ward KW, Kopple KD (2002) Molecular properties that influence the oral bioavailability of drug candidates. J Med Chem 45(12):2615-2623. https://doi.org/10.1021/jm02001 $7 n$

21. Athar M, Sona A, Bekono B, Ntie-Kang F (2019) Fundamental physical and chemical concepts behind "drug-likeness" and "natural product-likeness". Phys Sci Rev 4(12):20180101. https://doi.org/10.1515/psr-2018-0101

22. Ali I, Mukhtar SD, Hsieh MF, Alothman ZA, Alwarthan A (2018) Facile synthesis of indole heterocyclic compounds based micellar nano anticancer drugs. RSC Adv 8(66):37905-37914. https://doi.org/10.1039/c8ra 07060a

23. Chagas CM, Moss S, Alisaraie L (2018) Drug metabolites and their effects on the development of adverse reactions: Revisiting Lipinski's Rule of Five. Int J Pharm 549(1-2):133-149. https://doi.org/10.1016/j.ijpharm.2018.07.046

24. Huang $\mathrm{H}$, Chu CL, Chen L, Shui D (2019) Evaluation of potential inhibitors of squalene synthase based on virtual screening and in vitro studies. Comput Biol Chem 80:390-397. https://doi.org/10.1016/j. compbiolchem.2019.04.008

25. Lipinski CA (2004) Lead- and drug-like compounds: the rule-of-five revolution. Drug Discov Today Technol 1(4):337-341. https://doi.org/10.101 6/j.ddtec.2004.11.007

26. Tan DS (2004) Current progress in natural product-like libraries for discovery screening. Comb Chem High Throughput Screen 7(7):631-643. https://doi. org/10.2174/1386207043328418

27. Gleeson MP, Hersey A, Montanari D, Overington J (2011) Probing the links between in vitro potency, ADMET and physicochemical parameters. Nat Rev Drug Discov 10(3):197-208. https://doi.org/10.1038/nrd3367

28. Liu X, Testa B, Fahr A (2011) Lipophilicity and its relationship with passive drug permeation. Pharm Res 28(5):962-977. https://doi.org/10.1007/s11095010-0303-7

29. Ertl P, Rohde B, Selzer P (2000) Fast calculation of molecular polar surface area as a sum of fragment-based contributions and its application to the prediction of drug transport properties. J Med Chem 43(20):3714-3717. https://doi.org/10.1021/jm000942e

30. Li S, He H, Parthiban LJ, Yin H, Serajuddin AT (2005) IV-IVC considerations in the development of immediate-release oral dosage form. J Pharm Sci 94(7): 1396-1417. https://doi.org/10.1002/jps.20378

31. Strazielle N, Ghersi-Egea JF (2005) Factors affecting delivery of antiviral drugs to the brain. Rev Med Virol 15(2):105-133. https://doi.org/10.1002/ rmv.454

32. Prasanna S, Doerksen R (2009) Topological polar surface area: a useful descriptor in 2D-QSAR. Curr Med Chem 16(1):21-41. https://doi.org/10.2174/ 092986709787002817

33. Maximo da Silva M, Comin M, Santos Duarte T, Foglio M, de Carvalho J, do Carmo Vieira M, Nazari Formagio A (2015) Synthesis, antiproliferative activity and molecular properties predictions of galloyl derivatives. Molecules 20(4): 5360-5373. https://doi.org/10.3390/molecules20045360
34. Deosarkar SD, Pawar MP, Sawale RT, Hardas AR, Kalyankar TM (2015) Solvent effects on molar refraction and polarizability of 4-amino-5-chloro-N-(2(diethylamino)ethyl)-2 methoxybenzamide hydrochloride hydrate solutions at $30^{\circ} \mathrm{C}$. J Chem Pharmaceut Res 7(5):1107-1110

35. Banik I, Roy MN (2012) Study of solute-solvent interaction of some bioactive solutes prevailing in aqueous ascorbic acid solution. J Mol Liq 169:814. https://doi.org/10.1016/j.molliq.2012.03.006

36. Chen CP, Ahlers HW, Scott Dotson G, Lin YC, Chang WC, Maier A, Gadagbui B (2011) Efficacy of predictive modeling as a scientific criterion in dermal hazard identification for assignment of skin notations. Regul Toxicol Pharmacol 61(1):63-72. https://doi.org/10.1016/j.yrtph.2011.05.013

37. Dotson GS, Chen CP, Gadagbui B, Maier A, Ahlers HW, Lentz TJ (2011) The evolution of skin notations for occupational risk assessment: a new NIOSH strategy. Regul Toxicol Pharmacol 61(1):53-62. https://doi.org/10.1016/j. yrtph.2011.06.002

38. Gaur R, Thakur JP, Yadav DK, Kapkoti DS, Verma RK, Gupta N, Khan F, Saikia D, Bhakuni RS (2015) Synthesis, antitubercular activity, and molecular modeling studies of analogues of isoliquiritigenin and liquiritigenin, bioactive components from Glycyrrhiza glabra. Med Chem Res 24(9):34943503. https://doi.org/10.1007/s00044-015-1401-1

39. Green AK, Haley SL, Denise Dearing M, Barnes DM, Karasov WH (2004) Intestinal capacity of P-glycoprotein is higher in the juniper specialist, Neotoma stephensi, than the sympatric generalist. Neotoma Albigula 139(3): 325-333. https://doi.org/10.1016/j.cbpb.2004.09.017

\section{Publisher's Note}

Springer Nature remains neutral with regard to jurisdictional claims in published maps and institutional affiliations.

\section{Submit your manuscript to a SpringerOpen ${ }^{\circ}$ journal and benefit from:}

- Convenient online submission

- Rigorous peer review

- Open access: articles freely available online

High visibility within the field

- Retaining the copyright to your article

Submit your next manuscript at $>$ springeropen.com 